\title{
ABSOLUTE PROPER AND SPACE MOTION OF THE GLOBULAR CLUSTER M 2
}

\author{
M. GEFFERT ${ }^{1}$, C. FORNER ${ }^{1}$, M. HIESGEN ${ }^{2}$ and A.R. KLEMOLA ${ }^{3}$ \\ 1 Observatorium Hoher List, Sternwarte der Universität Bonn, W-5568 Daun, \\ Germany \\ ${ }^{2}$ Astronomisches Institut Münster, Wilhelm-Klemme Strasse 10, W-4400 Münster, \\ Germany \\ ${ }^{3}$ Lick Observatory, Santa Cruz, California 95064
}

\section{Introduction}

Space motions of globular clusters are important for the understanding of the kinematics and chemical evolution of the Milky Way. The greatest problem for the determination of the space motions of the globular clusters arises from the difficulties of getting absolute proper motions. In the past these were determined using classical stellar reference frames, modelling of the noncluster stars in the field and extragalactic objects in the region of the cluster (see e.g. Cudworth \& Hanson 1993; Geffert et al. 1993 for references). However, as shown for M 15 and M 3 (Geffert et al. 1993; Tucholke et al. 1993), the results based on different methods for deriving the absolute proper motions differ by up to $0.6 / 100$ yrs. In order to get a more complete view of the differences between the various methods it is therefore very interesting to get absolute proper motions based on all three methods for further objects. We present here the first results of a new proper motion study of the globular cluster M 2 .

\section{Plates, Measurements and Reduction}

12 plates with epochs ranging from 1902 to 1988 of our $(D=0.3 \mathrm{~m}, \mathrm{f}=5.13 \mathrm{~m})$ double refractor from Bonn (now located at Hoher List Observatory) were scanned with the PDS in Münster. We were able to derive positions and proper motions of 775 stars in a region of $1.5 \times 1.5$ degrees around the cluster centre. The errors of the proper motion components show a median accuracy of better than 0."1/100 yrs. The data were reduced with respect to the PPM catalogue. In order to get proper motions based on a larger number of reference stars we have determined a catalogue of 60 stars in the region of M 2 based on measurements of ESO Schmidt plates and the published positions of the Carte du Ciel project. This catalogue was based on 53 reference stars from the PPM catalogue while only 10 reference stars of the PPM were usable on the refractor plates. 


\section{The Orbit of $\mathbf{M} 2$}

From our data we obtained a preliminary absolute proper motion of M 2 of $-0.13 / 100$ yrs in $\alpha$ and $-0.68 / 100$ yrs in $\delta$. The comparison of these results with the ones obtained by Cudworth \& Hanson (1993) show differences (in the sense our minus other) of $-0.68 / 100$ yrs in $\alpha$ and $-0.26 / 100$ yrs in $\delta$. In order to demonstrate the influence of these differences in proper motions on the orbit of $M 2$, we have calculated orbits of $M 2$ based on the data of Cudworth \& Hanson (1993) and our data using the methods of Allen \& Martos (1988) and Odenkirchen (1991). Our data indicate a smaller orbit reaching only an $R_{\max }$ of about $17 \mathrm{kpc}$, while the orbit based on the data of Cudworth \& Hanson has an $R_{\max }$ of $30 \mathrm{kpc}$. The orbit calculation of Allen (1990) based on the earlier data of Cudworth \& Rauscher (1987) reaches even $60 \mathrm{kpc}$.
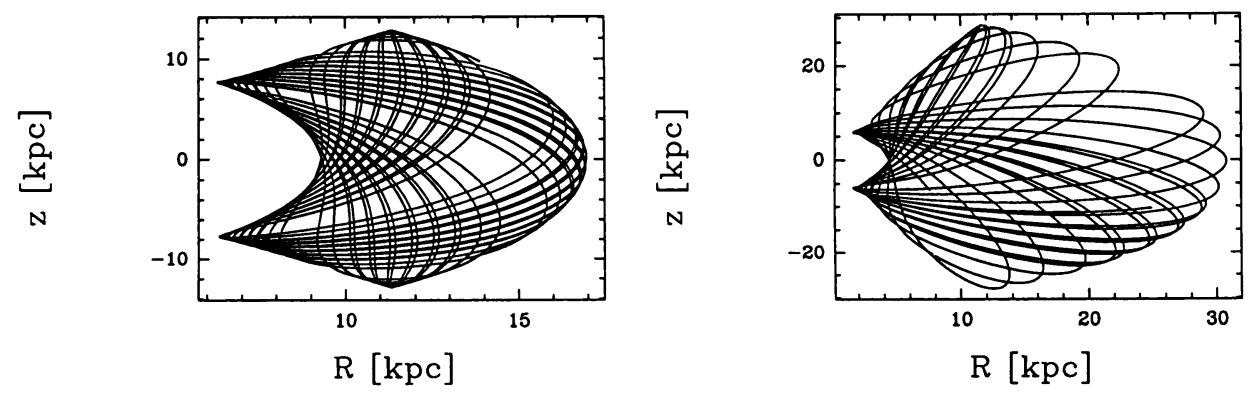

Figure 1. The meridional section of the orbit of $M 2$ based on our proper motions (left) and the ones obtained by Cudworth \& Hanson (1993) (right) integrated back over $10^{10}$ years.

\section{Future Aspects}

We plan to determine the absolute proper motion of $\mathbf{M} 2$ with respect to extragalactic objects. First, we will use absolute proper motions based on plates from the Lick Northern Proper Motion program and additional plates of the same telescope. Moreover, we are planning to use ESO Schmidt plates and glass copies of the POSS plates following the method first used by Geffert et al. (1993). Thus M 2 will be the first globular cluster with absolute proper motions being determined using two independent extragalactic calibrations. In addition, the absolute proper motions in the M 2 field may be used for the calibration of the HIPPARCOS proper motion system in the same way as pointed out by Geffert et al. (1993). 


\section{Acknowledgements}

We thank M. Odenkirchen (Bonn) for help with the orbit calculation. It is a pleasure to thank the Max-Planck-Institut für Astronomie in Heidelberg for letting us use their Palomar glass copies and their measuring engine.

\section{References}

Allen, C., 1990. RMxA, 20, 67.

Allen, C. and Martos, M.A., 1988. RMxA, 16, 25.

Cudworth, K.M. and Hanson, R.B., 1993. Astron. J., 105, 168.

Cudworth, K.M. and Rauscher, B.J., 1987. Astron. J., 93, 856.

Geffert, M., Colin, J., LeCampion, J.-F. and Odenkirchen, M., 1993. Astron. J., 106, 168.

Odenkirchen, M., 1991. Diploma Thesis, University of Bonn.

Tucholke, H.-J., Scholz, R.-D. and Brosche, P., 1993. Astron. Astrophys. Suppl., in press. 